\title{
Decentralized robust disturbance attenuation for a class of large-scale nonlinear systems
}

\author{
Yi Guo $^{\mathrm{a}, *}$, Zhong-Ping Jiang ${ }^{\mathrm{b}}$, David J. Hill ${ }^{\mathrm{a}}$ \\ ${ }^{a}$ School of Electrical and Information Engineering, The University of Sydney, Sydney, NSW 2006, Australia \\ ${ }^{\mathrm{b}}$ Department of Electrical Engineering, Polytechnic University, Six Metrotech Center, Boorklyn, NY 11201, USA
}

Received 4 June 1998; received in revised form 2 December 1998; accepted 21 January 1999

\begin{abstract}
We solve the problem of decentralized $H_{\infty}$ almost disturbance decoupling for a class of large-scale nonlinear uncertain systems in the absence of matching conditions. The method combines ideas from decentralized adaptive control and centralized nonlinear $H_{\infty}$ control. We relax earlier assumptions on the uncertain time-varying interconnections which are demanded to be only bounded by general nonlinear functions in this work. (c) 1999 Elsevier Science B.V. All rights reserved.
\end{abstract}

Keywords: Large-scale systems; Nonlinear systems; Decentralized control; Disturbance attenuation; Backstepping

\section{Introduction}

The study of large-scale systems has been motivated by their wide applicability to many practical systems such as power systems and spacecraft. Decentralized control schemes, whose design can be based on available local subsystem information and local performance criteria, are widely used - see [19] for instance. In this paper, we design the decentralized control law for a class of large-scale nonlinear systems whose normal forms exhibit triangular dependence on local coordinates. The objective of the design is to make the closed-loop interconnected system asymptotically stable while attenuating the effect of the disturbance.

Since various kinds of uncertainty commonly appear in the system models, either in the isolated subsystems or in the interconnections, adaptive and/or robust control of large-scale systems has received considerable attention during the last two decades. There are many control results concerning linear nominal systems with linear subsystem interconnections or interconnections satisfying linear bounds - see $[19,1,3,6,21,22,5,16]$ and references cited therein. While most of the results need matching condition assumptions, [16] presents a totally decentralized control scheme for systems of arbitrary relative degree. In [21], avoidance of matching conditions is achieved by applying integrator backstepping. Note also that interconnections are commonly required to be not only linear, but also weak in the sense that they are dominated by subsystem stability margins $[19,3,6,22,5]$. An advance on the interconnection constraint was presented in $[17,18]$ where the interconnection was extended to be bounded by any higher-order polynomial. Then in [4], a stabilizing design is given for a nonlinear time-varying system with the interconnection bounded by a general nonlinear function.

\footnotetext{
* Corresponding author.
} 
However, the uncertainties in $[17,18,4]$ require matching conditions. Recently in [9], based on the work of $[17,18]$ and backstepping and tuning function design [12], a class of large-scale nonlinear systems in their decentralized strict feedback form was designed to be adaptively stable; the matching condition was relaxed and the time-invariant interconnections are bounded by higher-order polynomials. In this paper, we assume time-varying interconnections without matching conditions which are bounded by general nonlinear functions and develop a robust control design.

In contrast to the large number of decentralized adaptive control results, there are few results on decentralized $H_{\infty}$ control. In [9,24,2,10], the decentralized linear $H_{\infty}$ control technique was applied for linear interconnected systems with linear interconnections where disturbances preserve the interconnection structure. Centralized nonlinear $H_{\infty}$ control has achieved considerable development since the initial result [20]. Especially in $[14,8,10]$, a triangular system structure was exploited to explicitly construct the controller which overcomes the general difficulty of solving the Hamilton-Jacobi-type partial differential inequality. The so-called "nonlinear $H_{\infty}$ almost disturbance decoupling problem" is solved in the sense that the designed internally stable system maintains arbitrary $\mathscr{L}_{2}$ gain from the disturbance input to output.

In this paper, we move to close the gap between decentralized and centralized nonlinear $H_{\infty}$ control. We consider the decentralized robust $H_{\infty}$ control problem for large-scale nonlinear systems and are motivated by [9]. The results combine the above-mentioned ideas from decentralized adaptive control and centralized nonlinear $H_{\infty}$ control for structured systems. Uncertain interconnections are extended to be time-varying and bounded by general nonlinear functions. Disturbances enter each subsystem affinely with subsystem states interacting through their input vector fields. The stabilizer design consists of constructing a nonlinear storage function [23] and the interactions are effectively dominated by subsystem stability margins. A decentralized control law is recursively constructed and the internally stable closed-loop system maintains any given $\mathscr{L}_{2}$-gain from the disturbance input to the output.

The remainder of the paper is organized as follows. In Section 2, we give the system configuration and state the main problem and necessary assumptions. Then in Section 3, our main theorem is presented and a recursive design procedure is developed to obtain the explicit decentralized controller. In Section 4, simulations for a two-subsystem example are shown to demonstrate the performance of our controller. Finally the paper is concluded by brief remarks in Section 5 .

Notations. The notation used in this paper is standard. $|\cdot|$ denotes the usual Euclidean norm for vectors. $\nabla f$ denotes the gradient $\partial f / \partial s$ of a real-valued function $f$. We say that $z:(0, T) \rightarrow \mathfrak{R}^{k}$ is in $\mathscr{L}_{2}(0, T)$ if $\int_{0}^{T}|z(t)|^{2} \mathrm{~d} t<\infty$. A continuous function $\phi: \mathfrak{R}_{+} \rightarrow \mathfrak{R}_{+}$is said to be of class $\mathscr{K}_{\infty}$ if it is strictly increasing and satisfies $\phi(0)=0$, and $\phi(s) \rightarrow \infty$ as $s \rightarrow \infty$. $\lambda_{\max }(P)$ and $\lambda_{\min }(P)$ denote the maximum and the minimum eigenvalue of any square matrix $P$. The arguments of a function will be omitted sometimes in the analysis when no confusion can arise.

\section{Problem formulation}

We consider in this paper a class of large-scale nonlinear systems $S$ which are composed of the following single-input-single-output ( $\mathrm{SISO})$ subsystems $S_{i}(1 \leqslant i \leqslant N)$ :

$$
\begin{aligned}
\dot{z}_{i} & =\left[\begin{array}{cccc}
0 & 1 & & 0 \\
& & \ddots & \\
0 & 0 & & 1 \\
0 & 0 & \ldots & 0
\end{array}\right]\left[\begin{array}{l}
z_{i 1} \\
\vdots \\
z_{i, \kappa-1} \\
z_{i \kappa}
\end{array}\right]+\left[\begin{array}{l}
0 \\
\vdots \\
0 \\
1
\end{array}\right] \cdot\left\{\xi_{i 1}+\gamma_{i 0}\left(t, z_{1}, \ldots, z_{N}\right)+p_{i 0}\left(t, z_{1}, \ldots, z_{N}\right) \omega_{i}\right\} \\
& \triangleq A_{i} z_{i}+B_{i} \cdot\left[\xi_{i 1}+\gamma_{i 0}\left(t, z_{1}, \ldots, z_{N}\right)+p_{i 0}\left(t, z_{1}, \ldots, z_{N}\right) \omega_{i}\right], \\
\dot{\xi}_{i 1} & =\xi_{i 2}+\gamma_{i 1}\left(t, z_{1}, \ldots, z_{N}, \xi_{i 1}\right)+p_{i 1}\left(t, z_{1}, \ldots, z_{N}, \xi_{i 1}\right) \omega_{i}, \\
\dot{\xi}_{i 2} & =\xi_{i 3}+\gamma_{i 2}\left(t, z_{1}, \ldots, z_{N}, \xi_{i 1}, \xi_{i 2}\right)+p_{i 2}\left(t, z_{1}, \ldots, z_{N}, \xi_{i 1}, \xi_{i 2}\right) \omega_{i},
\end{aligned}
$$




$$
\begin{aligned}
& \dot{\xi}_{i, n-\kappa}=u_{i}+\gamma_{i, n-\kappa}\left(t, z_{1}, \ldots, z_{N}, \xi_{i}\right)+p_{i, n-\kappa}\left(t, z_{1}, \ldots, z_{N}, \xi_{i}\right) \omega_{i}, \\
& y_{i}=z_{i 1}
\end{aligned}
$$

where $z_{i}=\left(z_{i 1}, \ldots, z_{i \kappa}\right) \in \mathfrak{R}^{\kappa}, z=\left(z_{1}^{\mathrm{T}}, \ldots, z_{N}^{\mathrm{T}}\right)^{\mathrm{T}}, \xi_{i}=\left(\xi_{i 1}, \ldots, \xi_{i, n-\kappa}\right) \in \mathfrak{R}^{n-\kappa}$, and $\xi=\left(\xi_{1}^{\mathrm{T}}, \ldots, \xi_{N}^{\mathrm{T}}\right)^{\mathrm{T}}, z$ and $\xi$ are the state vectors, $u_{i} \in \mathfrak{R}$ is the control input, $\omega_{i} \in \mathfrak{R}^{m_{i}}$ is the disturbance input, $y_{i} \in \mathfrak{R}$ is the to-be-controlled output; the unknown functions $\gamma_{i l}, p_{i l},(0 \leqslant l \leqslant n-\kappa)$ are locally Lipschitz in states and piecewise continuous in $t$, and $\gamma_{i l}(t, 0,0, \ldots, 0)=0$.

Our class of systems (1) is motivated by the time-invariant decentralized strict feedback form in [9] where necessary and sufficient geometric conditions are given to characterize a subclass of systems (1) (see [7]). Disturbances are introduced to enter each subsystem affinely with subsystem states interacting through their input vector fields. Furthermore, we allow in this paper for a larger set of nonlinear interconnections, as shown in the following assumptions.

Assumption 1. There exist known smooth functions $a_{i l j}(\cdot), b_{i l j}(\cdot), \varphi_{i l j}(\cdot), \Phi_{i l j}(\cdot)$, with $\varphi_{i l j}(0)=0, \Phi_{i l j}(0)=0$, such that for each $0 \leqslant l \leqslant n-\kappa$ the uncertain interconnections satisfy

$$
\begin{aligned}
& \left|\gamma_{i l}\left(t, z_{1}, \ldots, z_{N}, \xi_{i 1}, \ldots, \xi_{i l}\right)-\gamma_{i l}\left(t, 0, \ldots, 0, \xi_{i 1}, \ldots, \xi_{i l}\right)\right| \leqslant \sum_{j=1}^{N} a_{i l j}\left(\xi_{i 1}, \ldots, \xi_{i l}\right) \varphi_{i l j}\left(\left|z_{j}\right|\right), \\
& \left|p_{i l}\left(t, z_{1}, \ldots, z_{N}, \xi_{i 1}, \ldots, \xi_{i l}\right)-p_{i l}\left(t, 0, \ldots, 0, \xi_{i 1}, \ldots, \xi_{i l}\right)\right| \leqslant \sum_{j=1}^{N} b_{i l j}\left(\xi_{i 1}, \ldots, \xi_{i l}\right) \Phi_{i l j}\left(\left|z_{j}\right|\right) .
\end{aligned}
$$

For the sake of simplicity, we let $a_{i 0 j}=b_{i 0 j}=1$.

Assumption 2. There exist known smooth functions $\psi_{l}(\cdot), \Psi_{i l}(\cdot)$, with $\psi_{l}(0)=0$, such that for each $0 \leqslant l \leqslant n-\kappa$,

$$
\begin{aligned}
& \left|\gamma_{i l}\left(t, 0, \ldots, 0, \xi_{i 1}, \ldots, \xi_{i l}\right)\right| \leqslant \psi_{l}\left(\left|\left(\xi_{i 1}, \ldots, \xi_{i l}\right)\right|\right), \\
& \left|p_{i l}\left(t, 0, \ldots, 0, \xi_{i 1}, \ldots, \xi_{i l}\right)\right| \leqslant \Psi_{i l}\left(\left|\left(\xi_{i 1}, \ldots, \xi_{i l}\right)\right|\right) .
\end{aligned}
$$

Since $\gamma_{i 0}(t, 0)=0$, we can take $\psi_{0} \equiv 0$; and $\Psi_{i 0}$ is a nonnegative constant.

The objective of our design is to find robust decentralized controllers in terms of local subsystem states and make the whole interconnected system globally uniformly asymptotically stable while arbitrarily attenuating the effect of the disturbance in the sense of $\mathscr{L}_{2}$ gain. A precise statement of this control problem is given below:

Problem of decentralized $H_{\infty}$ almost disturbance decoupling. Find decentralized smooth state feedback controllers $u_{i}=u_{i}\left(z_{i}, \xi_{i}\right)$ such that, for any given positive constant $\mu$, the closed-loop interconnected system satisfies the following dissipation inequality

$$
\int_{0}^{T}|y|^{2} \mathrm{~d} t \leqslant \mu \int_{0}^{T}|w|^{2} \mathrm{~d} t+v(z(0), \xi(0)), \quad \forall \omega \in \mathscr{L}_{2}(0, T), \quad \forall T \geqslant 0,
$$

where $v$ is a positive semidefinite function and $(z(0), \xi(0))$ is the initial condition. Furthermore, the origin is globally uniformly asymptotically stable (GUAS) if $w=0$.

Remark 1. Nonlinear gain bounds on the interconnections are motivated by ideas used in recent stability results [13,11]. Nonlinear interconnection bounds with a matching condition were used in [9] where a saturation type of controller was designed to stabilize a class of uncertain time-varying nonlinear interconnected systems. 


\section{Main result}

The following theorem states our main result:

Theorem 1. Under Assumptions 1 and 2, the problem of decentralized $H_{\infty}$ almost disturbance decoupling is solvable for system (1).

The proof of Theorem 1 uses a combination of centralized $H_{\infty}$ almost disturbance decoupling method [14], its robust version [10] and decentralized designs [4,9]. A stepwise procedure is presented by application of robust backstepping to the large-scale system (1).

Proof Step 0: We start by considering the $z_{i}$-subsystem with $\xi_{i 1}$ as the virtual control input. Choose the storage function:

$$
V_{i}\left(z_{i}\right)=\phi_{i}\left(V_{i 0}\left(z_{i}\right)\right),
$$

where $\phi_{i}(\cdot)$ is a smooth $\mathscr{K}_{\infty}$ function and $V_{i 0}=z_{i}^{\mathrm{T}} P_{i} z_{i}$ with $P_{i}$ is a positive-definite symmetric matrix solving the algebric Riccati equation:

$$
A_{i}^{\mathrm{T}} P_{i}+P_{i} A_{i}-2 \epsilon P_{i} B_{i} B_{i}^{\mathrm{T}} P_{i}+Q_{i}=0,
$$

where $\epsilon_{i}$ is a positive constant and $Q_{i}$ is a positive-definite symmetric matrix.

Differentiating (7) along the solution of $z_{i}$-subsystem, we have

$$
\dot{V}_{i}=\frac{\partial \phi_{i}}{\partial V_{i 0}} \cdot 2 z_{i}^{\mathrm{T}} P_{i} \cdot\left\{A_{i} z_{i}+B_{i}\left[\xi_{i 1}+\left(\gamma_{i 0}(t, z)-\gamma_{i 0}(t, 0)\right)+\left(p_{i 0}(t, z)-p_{i 0}(t, 0)\right) \omega_{i}+p_{i 0}(t, 0) \omega_{i}\right]\right\} .
$$

Using the inequality $2 a b \leqslant a^{2}+b^{2},(a, b \in \mathfrak{R})$ and Assumption 1, the interactions in the second term in the square bracket of (9) are decoupled which gives

$$
\begin{aligned}
& \frac{\partial \phi_{i}}{\partial V_{i 0}} 2 z_{i}^{\mathrm{T}} P_{i} B_{i}\left(\gamma_{i 0}(t, z)-\gamma_{i 0}(t, 0)\right) \\
& \quad \leqslant\left|\frac{\partial \phi_{i}}{\partial V_{i 0}}\right| \cdot\left|2 z_{i}^{\mathrm{T}} P_{i} B_{i}\right| \sum_{j=1}^{N} \varphi_{i 0 j}\left(\left|z_{j}\right|\right) \\
& \quad \leqslant\left|\frac{\partial \phi_{i}}{\partial V_{i 0}}\right|^{2} \cdot\left|z_{i}^{\mathrm{T}} P_{i} B_{i}\right|^{2} \sum_{j=1}^{N} \delta_{j 0}+\sum_{j=1}^{N} \delta_{j 0}^{-1} \varphi_{i 0 j}^{2}\left(\left|z_{j}\right|\right) .
\end{aligned}
$$

Similarly, the third term in the square bracket of (9) turns to

$$
\begin{aligned}
& \frac{\partial \phi_{i}}{\partial V_{i 0}} 2 z_{i}^{\mathrm{T}} P_{i} B_{i}\left(p_{i 0}(t, z)-p_{i 0}(t, 0)\right) \omega_{i} \\
& \quad \leqslant \sum_{j=1}^{N} \frac{1}{\tau_{i}}\left|\frac{\partial \phi_{i}}{\partial V_{i 0}}\right|^{2} \cdot\left|z_{i}^{\mathrm{T}} P_{i} B_{i}\right|^{2} \Phi_{i 0 j}\left(\left|z_{j}\right|\right)^{2}+N \tau_{i}\left|\omega_{i}\right|^{2} \\
& \quad \leqslant \frac{1}{4 \tau_{i}^{2}}\left|\frac{\partial \phi_{i}}{\partial V_{i 0}}\right|^{4} \cdot\left|z_{i}^{\mathrm{T}} P_{i} B_{i}\right|^{4} \sum_{j=1}^{N} \varrho_{j 0}+\sum_{j=1}^{N} \varrho_{j 0}^{-1} \Phi_{i 0 j}\left(\left|z_{j}\right|\right)^{4}+N \tau_{i}\left|\omega_{i}\right|^{2},
\end{aligned}
$$

where $\delta_{j 0}, \varrho_{i 0}, \tau_{i}$ are any positive constants.

For the last term of (9), which is time-varying, we apply the bound from Assumption 2 to give

$$
\frac{\partial \phi_{i}}{\partial V_{i 0}} 2 z_{i}^{\mathrm{T}} P_{i} B_{i} p_{i 0}(t, 0) \omega_{i} \leqslant \frac{1}{d_{2 i}}\left|\frac{\partial \phi_{i}}{\partial V_{i 0}}\right|^{2}\left|z_{i}^{\mathrm{T}} P_{i} B_{i}\right|^{2} \Psi_{i 0}^{2}+d_{2 i}\left|\omega_{i}\right|^{2},
$$

where $d_{2 i}$ is any positive constant. 
Choose our virtual control $\xi_{i 1}=\xi_{i 1}^{*}\left(z_{i}\right)$ as

$$
\begin{aligned}
\xi_{i 1}^{*}\left(z_{i}\right)= & -\left\{\epsilon_{i} B_{i}^{\mathrm{T}} P_{i} z_{i}+\frac{1}{2} \frac{\partial \phi_{i}}{\partial V_{i 0}}\left(z_{i}^{\mathrm{T}} P_{i} B_{i}\right)\left(\sum_{j=1}^{N} \delta_{j 0}\right)+\frac{1}{8 \tau_{i}^{2}}\left(\frac{\partial \phi_{i}}{\partial V_{i 0}}\right)^{3}\left(z_{i}^{\mathrm{T}} P_{i} B_{i}\right)^{3}\left(\sum_{j=1}^{N} \varrho_{j 0}\right)\right. \\
& \left.+\frac{1}{2 d_{2 i}}\left(\frac{\partial \phi_{i}}{\partial V_{i 0}}\right)\left(z_{i}^{\mathrm{T}} P_{i} B_{i}\right) \Psi_{i 0}^{2}\right\} .
\end{aligned}
$$

Notice $\xi_{i 1}^{*}(0)=0$.

When substituting (13) into (9) and applying the bounds (10)-(12), we get

$$
\begin{aligned}
\dot{V}_{i} & \leqslant \frac{\partial \phi_{i}}{\partial V_{i 0}}\left(-z_{i}^{\mathrm{T}} Q_{i} z_{i}\right)+\left[\sum_{j=1}^{N} \delta_{i 0}^{-1} \varphi_{i 0 j}^{2}\left(\left|z_{j}\right|\right)+\sum_{j=1}^{N} \varrho_{j 0}^{-1} \Phi_{i 0 j}^{4}\left(\left|z_{j}\right|\right)\right]+\left(N \tau_{i}+d_{2 i}\right)\left|\omega_{i}\right|^{2} \\
& \triangleq-\alpha_{i 0}\left(\left|z_{i}\right|\right)+\sum_{j=1}^{N} \beta_{i 0 j}\left(\left|z_{j}\right|\right)+\gamma_{i 0}\left|\omega_{i}\right|^{2} .
\end{aligned}
$$

By constructing virtual control $\xi_{i 1}$ according to (13) for the $z_{i}$-subsystem, we achieve a dissipation inequality (14) which implies that nett dissipation is determined by the dominance of a local state function $\alpha_{i 0}$ over a state function $\left(\sum_{j=1}^{N} \beta_{i 0 j}\right)$. Note that inequality (14) is only valid when $\xi_{i 1}=\xi_{i 1}^{*}$; otherwise, additional terms must be included. We leave the choice of $\phi_{i}$ till the final stage of the design problem. From here, we start our recursive backstepping procedure.

Step 1: Augment the $z_{i}$-subsystem with the $\xi_{i 1}$-subsystem, and choose a storage function as

$$
W_{i 1}\left(z_{i}, \xi_{i 1}\right)=V_{i}\left(z_{i}\right)+\left(\xi_{i 1}-\xi_{i 1}^{*}\right)^{2} .
$$

Denote

$$
\begin{aligned}
-\dot{\xi}_{i 1}^{*} & =-\frac{\partial \xi_{i 1}^{*}}{\partial z_{i}}\left(A_{i} z_{i}+B_{i} \xi_{i 1}\right)-\frac{\partial \xi_{i 1}^{*}}{\partial z_{i}} B_{i}\left[\gamma_{i 0}(t, z)+p_{i 0}(t, z) \omega_{i}\right] \\
& \triangleq \vartheta_{i 1}\left(z_{i}, \xi_{i 1}\right)+\sigma_{i 0}\left(z_{i}\right)\left[\gamma_{i 0}(t, z)+p_{i 0}(t, z) \omega_{i}\right]
\end{aligned}
$$

Differentiating $W_{i 1}$ along the solutions of the $\left(z_{i}, \xi_{i 1}\right)$-subsystem yields

$$
\begin{aligned}
\dot{W}_{i 1} \leqslant & -\alpha_{i 0}\left(\left|z_{i}\right|\right)+\sum_{j=1}^{N} \beta_{i 0 j}\left(\left|z_{j}\right|\right)+\gamma_{i 0}\left|\omega_{i}\right|^{2}+2 \widetilde{\xi}_{i 1}\left\{\xi_{i 2}+\left(\frac{\partial \phi_{i}}{\partial V_{i 0}} z_{i}^{\mathrm{T}} P_{i} B_{i}+\vartheta_{i 1}\right)\right. \\
& +\sum_{k=0}^{1} \sigma_{i k}\left[\gamma_{i k}\left(t, z, \xi_{i 1}\right)-\gamma_{i k}\left(t, 0, \xi_{i 1}\right)\right]+\sum_{k=0}^{1} \sigma_{i k}\left[p_{i k}\left(t, z, \xi_{i 1}\right)-p_{i k}\left(t, 0, \xi_{i 1}\right)\right] \omega_{i} \\
& \left.+\gamma_{i 1}\left(t, 0, \xi_{i 1}\right)+\sum_{k=0}^{1} \sigma_{i k} p_{i k}\left(t, 0, \xi_{i 1}\right) \omega_{i}\right\}
\end{aligned}
$$

where we denote $\widetilde{\xi}_{i 1}=\xi_{i 1}-\xi_{i 1}^{*}\left(z_{i}\right)$ and $\sigma_{i 1}=1$.

Since $\xi_{i 1}^{*}\left(z_{i}\right)$ is smooth and $\xi_{i 1}^{*}(0)=0$, there exists a smooth function $\zeta_{i}\left(z_{i}\right)$ such that $\xi_{i 1}^{*}\left(z_{i}\right)=z_{i}^{\mathrm{T}} \zeta_{i}\left(z_{i}\right)$. This together with the definition of $\widetilde{\xi}_{i 1}$ gives

$$
\left|\xi_{i 1}\right| \leqslant \widetilde{\zeta}_{i}\left(z_{i}\right) \cdot\left(\left|\widetilde{\xi}_{i 1}\right|+\left|z_{i}\right|\right)
$$

where $\widetilde{\zeta}_{i}\left(z_{i}\right)$ is a smooth nonnegative function. 
Applying the bounds from Assumption 2 to the second last term of (17), and using (18) and the fact that $\psi_{i 1}$ is smooth and $\psi_{i 1}(0)=0$, we have

$$
\begin{aligned}
2 \widetilde{\xi}_{i 1} \gamma_{i 1}\left(t, 0, \xi_{i 1}\right) & \leqslant 2\left|\widetilde{\xi}_{i 1}\right| \cdot \psi_{i 1}\left(\left|\xi_{i 1}\right|\right) \\
& \leqslant 2\left|\widetilde{\xi}_{i 1}\right| \bar{\psi}_{i 1}\left(\left|\xi_{i 1}\right|\right)\left|\xi_{i 1}\right| \\
& \leqslant 2\left|\widetilde{\xi}_{i 1}\right| \widetilde{\psi}_{i 1}\left(z_{i}, \xi_{i 1}\right)\left(\left|\widetilde{\xi}_{i 1}\right|+\left|z_{i}\right|\right) \\
& \leqslant 2\left|\widetilde{\xi}_{i 1}\right|^{2} \widetilde{\psi}_{i 1}\left(z_{i}, \xi_{i 1}\right)+\left.\frac{1}{d_{1 i}} \widetilde{\xi}_{i 1}\right|^{2} \widetilde{\psi}_{i 1}^{2}\left(z_{i}, \xi_{i 1}\right)+d_{1 i}\left|z_{i}\right|^{2},
\end{aligned}
$$

where $d_{1 i}$ is a positive design parameter to be given later and $\widetilde{\psi}_{i 1}$ is a smooth nonnegative function.

Similarly, applying Assumption 2, for any $d_{2 i}>0$, the last term of (17) turns to

$$
\begin{aligned}
2 \widetilde{\xi}_{i 1} & \sum_{k=0}^{1} \sigma_{i k} p_{i k}\left(t, 0, \xi_{i 1}\right) \omega_{i} \\
& \leqslant \frac{1}{d_{2 i}}\left|\widetilde{\xi}_{i 1}\right|^{2}\left[\sum_{k=0}^{1} \sigma_{i k} \Psi_{i k}\left(\left|\xi_{i k}\right|\right)\right]^{2}+d_{2 i}\left|\omega_{i}\right|^{2} .
\end{aligned}
$$

Applying the bounds from Assumption 1 to the fourth and third last terms of (17), respectively, we obtain

$$
\begin{aligned}
& 2 \widetilde{\xi}_{i 1} \sum_{k=0}^{1} \sigma_{i k}\left[\gamma_{i k}\left(t, z, \xi_{i 1}\right)-\gamma_{i k}\left(t, 0, \xi_{i 1}\right)\right] \\
& \quad \leqslant 2\left|\widetilde{\xi}_{i 1}\right| \sum_{k=0}^{1}\left|\sigma_{i k}\right| \sum_{j=1}^{N} a_{i k j}\left(\xi_{i 1}\right) \varphi_{i k j}\left(\left|z_{j}\right|\right) \\
& \quad \leqslant\left|\widetilde{\xi}_{i 1}\right|^{2} \sum_{k=0}^{1}\left|\sigma_{i k}\right|^{2} \sum_{j=1}^{N} \delta_{j k} a_{i k j}^{2}\left(\xi_{i 1}\right)+\sum_{k=0}^{1} \sum_{j=1}^{N} \delta_{j k}^{-1} \varphi_{i k j}^{2}\left(\left|z_{j}\right|\right) \\
& 2 \widetilde{\xi}_{i 1} \sum_{k=0}^{1} \sigma_{i k}\left[p_{i k}\left(t, z, \xi_{i 1}\right)-p_{i k}\left(t, 0, \xi_{i 1}\right)\right] \omega_{i} \\
& \quad \leqslant 2\left|\widetilde{\xi}_{i 1}\right| \sum_{k=0}^{1}\left|\sigma_{i k}\right| \sum_{j=1}^{N} b_{i k j}\left(\xi_{i 1}\right) \Phi_{i k j}\left(\left|z_{j}\right|\right)\left|\omega_{i}\right| \\
& \quad \leqslant \frac{1}{4 \tau_{i}^{2}}\left|\widetilde{\xi}_{i 1}\right|^{4} \sum_{k=0}^{1}\left|\sigma_{i k}\right|^{4} \sum_{j=1}^{N} \varrho_{j k} b_{i k j}^{4}\left(\xi_{i 1}\right)+\sum_{k=0}^{1} \sum_{j=1}^{N} \varrho_{j k}^{-1} \Phi_{i k j}^{4}\left(\left|z_{j}\right|\right)+2 N \tau_{i}\left|\omega_{i}\right|^{2},
\end{aligned}
$$

where $\delta_{i k}, \varrho_{i k}$ are positive constants.

Now, we can choose the virtual controller $\xi_{i 2}=\xi_{i 2}^{*}$ as

$$
\begin{aligned}
\xi_{i 2}^{*}= & -\frac{1}{2}\left\{c_{i 1} \widetilde{\xi}_{i 1}+\frac{\partial \phi_{i}}{\partial V_{i 0}} 2 z_{i}^{\mathrm{T}} P_{i} B_{i}+2 \vartheta_{i 1}+2 \widetilde{\xi}_{i 1} \widetilde{\psi}_{i 1}\left(z_{i}, \xi_{i 1}\right)+\frac{1}{d_{1 i}} \widetilde{\xi}_{i 1} \widetilde{\psi}_{i 1}^{2}\left(z_{i}, \xi_{i 1}\right)\right. \\
& \left.+\frac{1}{d_{2 i}} \widetilde{\xi}_{i 1}\left[\sum_{k=0}^{1} \sigma_{i k} \Psi_{i 1}\left(\left|\xi_{i k}\right|\right)\right]^{2}+\widetilde{\xi}_{i 1} \sum_{k=0}^{1} \sigma_{i k}^{2} \sum_{j=1}^{N} \delta_{j k} a_{i k j}^{2}\left(\xi_{i 1}\right)+\frac{1}{4 \tau_{i}^{2}} \widetilde{\xi}_{i 1}^{3} \sum_{k=0}^{1} \sigma_{i k}^{4} \sum_{j=1}^{N} \varrho_{j k} b_{i k j}^{4}\left(\xi_{i 1}\right)\right\},
\end{aligned}
$$

where $c_{i 1}$ is a positive constant. 
Then (17) with the bounds (19)-(22) implies

$$
\dot{W}_{i 1} \leqslant-\alpha_{i 1}\left(\left|z_{i}\right|\right)-C_{i 11} \widetilde{\xi}_{i 1}^{2}+\sum_{j=1}^{N} \beta_{i 1 j}\left(\left|z_{j}\right|\right)+\gamma_{i 1}\left|\omega_{i}\right|^{2},
$$

where

$$
\begin{aligned}
& \alpha_{i 1}\left(\left|z_{i}\right|\right)=\alpha_{i 0}\left(\left|z_{i}\right|\right)-d_{1 i}\left|z_{i}\right|^{2}, \\
& \beta_{i 1 j}\left(\left|z_{j}\right|\right)=\beta_{i 0 j}+\sum_{k=0}^{1}\left\{\delta_{j k}^{-1} \varphi_{i k j}^{2}\left(\left|z_{j}\right|\right)+\varrho_{j k}^{-1} \Phi_{i k j}^{4}\left(\left|z_{j}\right|\right)\right\}, \\
& \gamma_{i 1}=\gamma_{i 0}+2 N \tau_{i}+d_{2 i}, \\
& C_{i 11}=c_{i 1} .
\end{aligned}
$$

Note that inequality (24) is only valid when $\xi_{i 2}=\xi_{i 2}^{*}$.

So from Step 1 the dissipation inequality (24) possesses the same property as described at the end of Step 0 with modified bound functions. We now show that this property remains while recursively argumenting the $\xi_{i l}$-subsystem.

From Step 2 to Step $n-\kappa$, we can establish the following Claim (see the appendix for the proof).

Claim. Given any index $2 \leqslant l \leqslant n-\kappa$, for the system $S_{i l}$

$$
\begin{aligned}
& \dot{z}_{i}=A_{i} z_{i}+B_{i}\left[\xi_{i 1}+\gamma_{i 0}(t, z)+p_{i 0}(t, z) \omega_{i}\right], \\
& \dot{\xi}_{i 1}=\xi_{i 2}+\gamma_{i 1}\left(t, z, \xi_{i 1}\right)+p_{i 1}\left(t, z, \xi_{i 1}\right) \omega_{i}, \\
& \quad \vdots \\
& \dot{\xi}_{i l}=\xi_{i, l+1}+\gamma_{i l}\left(t, z, \xi_{i 1}, \ldots, \xi_{i l}\right)+p_{i l}\left(t, z, \xi_{i 1}, \ldots, \xi_{i l}\right) \omega_{i},
\end{aligned}
$$

there exist $\imath+1$ smooth functions

$$
\xi_{i k}^{*}=\xi_{i k}^{*}\left(z_{i}, \xi_{i 1}, \ldots, \xi_{i, k-1}\right), \xi_{i k}^{*}(0,0, \ldots, 0)=0,1 \leqslant k \leqslant l+1
$$

such that in new coordinates

$$
\widetilde{z}_{i}=z_{i}, \widetilde{\xi}_{i k}=\xi_{i k}-\xi_{i k}^{*}\left(z_{i}, \xi_{i 1}, \ldots, \xi_{i, k-1}\right), \quad 1 \leqslant k \leqslant l,
$$

the storage function

$$
W_{i l}=V_{i}+\sum_{k=1}^{l} \widetilde{\xi}_{i k}^{2},
$$

has time derivative, with $\xi_{i, l+1}=\xi_{i, l+1}^{*}$, satisfying the dissipation inequality

$$
\dot{W}_{i l} \leqslant-\alpha_{i l}\left(\left|z_{i}\right|\right)-\sum_{k=1}^{l} C_{i l k} \widetilde{\xi}_{i k}^{2}+\sum_{j=1}^{N} \beta_{i l j}\left(\left|z_{j}\right|\right)+\gamma_{i l}\left|\omega_{i}\right|^{2},
$$

where

$$
\begin{aligned}
& \alpha_{i l}\left(\left|z_{i}\right|\right)=\alpha_{i, l-1}\left(\left|z_{i}\right|\right)-d_{1 i}\left|z_{i}\right|^{2} \\
& \beta_{i l j}\left(\left|z_{j}\right|\right)=\beta_{i, l-1, j}\left(\left|z_{j}\right|\right)+\sum_{k=0}^{l}\left\{\delta_{j k}^{-1} \varphi_{i k j}^{2}\left(\left|z_{j}\right|\right)+\varrho_{j k}^{-1} \Phi_{i k j}^{4}\left(\left|z_{j}\right|\right)\right\}, \\
& \gamma_{i l}=\gamma_{i, l-1}+(l+1) N \tau_{i}+d_{2 i},
\end{aligned}
$$




$$
\begin{aligned}
& C_{i l k}=C_{i, l-1, k}-d_{3 i} \quad \text { for } k=1, \ldots, \imath-1, \\
& C_{i l k}=c_{i k} \quad \text { for } k=\imath .
\end{aligned}
$$

As shown in Step 1, the Claim holds for the system (29) with $l=1$. At Step $n-\kappa$, by means of the Claim, a decentralized state feedback control

$$
u_{i}=\xi_{i, n-\kappa+1}^{*}\left(z_{i}, \xi_{i}\right)
$$

is iteratively built. For the closed-loop system $S_{i}$, if we denote the storage function as

$$
W_{i}\left(z_{i}, \xi_{i}\right) \triangleq W_{i, n-\kappa}\left(z_{i}, \xi_{i}\right)=V_{i}+\sum_{k=1}^{n-\kappa} \widetilde{\xi}_{i k}^{2},
$$

we have

$$
\dot{W}_{i} \leqslant-\alpha_{i}\left(\left|z_{i}\right|\right)-\sum_{l=1}^{n-\kappa} C_{i l} \widetilde{\xi}_{i l}^{2}+\sum_{j=1}^{N} \beta_{i j}\left(\left|z_{j}\right|\right)+\gamma_{i}\left|\omega_{i}\right|^{2}
$$

where

$$
\begin{aligned}
& \alpha_{i}\left(\left|z_{i}\right|\right)=\frac{\partial \phi_{i}}{\partial V_{i 0}}\left(z_{i}^{\mathrm{T}} Q_{i} z_{i}\right)-(n-\kappa) d_{1 i}\left|z_{i}\right|^{2}, \\
& \beta_{i j}\left(\left|z_{j}\right|\right)=\sum_{l=0}^{n-\kappa}\left\{(n-\kappa-l+1) \delta_{j l}^{-1} \varphi_{i l j}^{2}\left(\left|z_{j}\right|\right)+(n-\kappa-l+1) \varrho_{j l}^{-1} \Phi_{i l j}^{4}\left(\left|z_{j}\right|\right)\right\}, \\
& \gamma_{i}=(n-\kappa+1)\left[\frac{1}{2}(n-\kappa+2) N \tau_{i}+d_{2 i}\right], \\
& C_{i l}=c_{i l}-(n-\kappa-l) d_{3 i} .
\end{aligned}
$$

Choose the positive parameter $d_{3 i}$ as

$$
d_{3 i}<\min _{1 \leqslant l \leqslant n-\kappa-1}\left\{\frac{c_{i l}}{n-\kappa-l}\right\}
$$

so that $C_{i l}>0$.

Define a storage function for the whole interconnected system $S$ as

$$
W(z, \xi)=\sum_{i=1}^{N} W_{i}\left(z_{i}, \xi_{i}\right)
$$

Then from (38), we know

$$
\dot{W} \leqslant \sum_{i=1}^{N}\left\{-\alpha_{i}\left(\left|z_{i}\right|\right)-\sum_{l=1}^{n-\kappa} C_{i l} \widetilde{\xi}_{i l}^{2}+\sum_{j=1}^{N} \beta_{i j}\left(\left|z_{j}\right|\right)+\gamma_{i}\left|\omega_{i}\right|^{2}\right\} .
$$

The idea now is to compensate for the effect of the other subsystem interactions using the subsystem stability margins.

Notice

$$
\sum_{i=1}^{N} \sum_{j=1}^{N} \beta_{i j}\left(\left|z_{j}\right|\right)=\sum_{i=1}^{N} \sum_{j=1}^{N} \beta_{j i}\left(\left|z_{i}\right|\right) .
$$


For smooth functions vanishing at the origin $\varphi_{j l i}(\cdot)$ and $\Phi_{j l i}(\cdot)$, we have

$$
\begin{aligned}
& \varphi_{j l i}\left(\left|z_{i}\right|\right)=\left|z_{i}\right| \int_{0}^{1} \nabla \varphi_{j l i}\left(s\left|z_{i}\right|\right) \mathrm{d} s \leqslant\left|z_{i}\right| \widetilde{\varphi}_{j l i}\left(\left|z_{i}\right|\right), \\
& \Phi_{j l i}\left(\left|z_{i}\right|\right)=\left|z_{i}\right| \int_{0}^{1} \nabla \Phi_{j l i}\left(s\left|z_{i}\right|\right) \mathrm{d} s \leqslant\left|z_{i}\right| \widetilde{\Phi}_{j l i}\left(\left|z_{i}\right|\right),
\end{aligned}
$$

where $\widetilde{\varphi}_{j l i}\left(\left|z_{i}\right|\right), \widetilde{\Phi}_{j l i}\left(\left|z_{i}\right|\right)$ are smooth and nondecreasing functions.

Observe that

$$
\lambda_{\min }\left(P_{i}\right)\left|z_{i}\right|^{2} \leqslant V_{i 0}\left(z_{i}\right)
$$

which gives

$$
\left|z_{i}\right| \leqslant \sqrt{\frac{V_{i 0}\left(z_{i}\right)+1}{\lambda_{\min }\left(P_{i}\right)}} \triangleq \eta_{i}\left(V_{i 0}\right) .
$$

Note that the functions $\eta_{i}$ are smooth positive functions.

By the nondecreasing property of functions $\widetilde{\varphi}_{j l i}(\cdot)$ and $\widetilde{\Phi}_{j l i}(\cdot)$, we have

$$
\begin{aligned}
& \widetilde{\varphi}_{j l i}\left(\left|z_{i}\right|\right) \leqslant \widetilde{\varphi}_{j l i} \circ \eta_{i}\left(V_{i 0}\right), \\
& \widetilde{\Phi}_{j l i}\left(\left|z_{i}\right|\right) \leqslant \widetilde{\Phi}_{j l i} \circ \eta_{i}\left(V_{i 0}\right) .
\end{aligned}
$$

Now construct the derivative of $\phi_{i}\left(V_{i 0}\right)$ as

$$
\begin{aligned}
\frac{\partial \phi_{i}}{\partial\left(V_{i 0}\right)}= & k_{i}+\frac{1}{\lambda_{\min }\left(Q_{i}\right)} \sum_{j=1}^{N} \sum_{l=0}^{n-\kappa}(n-\kappa-l+1) \delta_{i l}^{-1}\left[\widetilde{\varphi}_{j l i} \circ \eta_{i}\left(V_{i 0}\right)\right]^{2} \\
& +\frac{V_{i 0}}{\lambda_{\min }\left(Q_{i}\right) \cdot \lambda_{\min }\left(P_{i}\right)} \cdot \sum_{j=1}^{N} \sum_{l=0}^{n-\kappa}(n-\kappa-l+1) \varrho_{i l}^{-1}\left[\widetilde{\Phi}_{j l i} \circ \eta_{i}\left(V_{i 0}\right)\right]^{4},
\end{aligned}
$$

where $k_{i}$ 's are positive constants. It can be checked that by the construction $(52)$ and $\phi_{i}(0)=0, \phi_{i}$ is a smooth $\mathscr{K}_{\infty}$ function.

Substitute (52) into (39) in view of (40), (46) and (51), and we have the dominance condition

$$
\sum_{i=1}^{N} \alpha_{i}\left(\left|z_{i}\right|\right) \geqslant \sum_{i=1}^{N} D_{i}\left|z_{i}\right|^{2}+\sum_{i=1}^{N} \sum_{j=1}^{N} \beta_{i j}\left(\left|z_{j}\right|\right),
$$

where $D_{i}=k_{i} \lambda_{\min }\left(Q_{i}\right)-(n-\kappa) d_{1 i}$. Choose the design parameter $d_{1 i}$ as

$$
d_{1 i}<\frac{k_{i} \lambda_{\min }\left(Q_{i}\right)}{n-\kappa}
$$

so that $D_{i}>0$.

Then from (45) and (53) we obtain

$$
\dot{W} \leqslant \sum_{i=1}^{N}\left\{-D_{i}\left|z_{i}\right|^{2}-\sum_{l=1}^{n-\kappa} C_{i l} \widetilde{\xi}_{i l}^{2}+\gamma_{i}\left|\omega_{i}\right|^{2}\right\}
$$

and it follows:

$$
\dot{W} \leqslant \sum_{i=1}^{N}\left\{-D_{i}\left|z_{i 1}\right|^{2}+\gamma_{i}\left|\omega_{i}\right|^{2}\right\} .
$$

Taking the integral along time $t$, the $\mathscr{L}_{2}$ gain from $\omega$ to $y$ of the closed-loop system is obtained as

$$
\int_{0}^{T}|y|^{2} \mathrm{~d} t \leqslant \mu \int_{0}^{T}|w|^{2} \mathrm{~d} t+v(z(0), \xi(0)),
$$


where

$$
\begin{aligned}
& \mu=\min _{1 \leqslant i \leqslant N}\left\{\gamma_{i}\right\} / \max _{1 \leqslant i \leqslant N}\left\{D_{i}\right\}, \\
& v(z(0), \xi(0))=W(z(0), \xi(0)) / \max _{1 \leqslant i \leqslant N}\left\{D_{i}\right\} .
\end{aligned}
$$

In the absence of the disturbance, i.e. when $\omega_{i}=0$, from (55), we have

$$
\dot{W} \leqslant \sum_{i=1}^{N}\left\{-D_{i}\left|z_{i}\right|^{2}-\sum_{l=1}^{n-\kappa} C_{i l} \widetilde{\xi}_{i l}^{2}\right\} \triangleq-W_{a}(z, \xi) \leqslant 0 .
$$

From (37) and (44),

$$
W(z, \xi)=\sum_{i=1}^{N}\left\{\phi_{i}\left(V_{i 0}\left(z_{i}\right)\right)+\sum_{l=1}^{n-\kappa} \widetilde{\xi}_{i l}^{2}\right\}
$$

is a continuously differentiable, positive definite and radially unbounded function and its derivative is negative definite. Hence we obtain the GUAS of the closed-loop system when $\omega_{i}=0$.

Remark 2. The result has points of connection with stability results for large-scale systems - see [15] for instance. We design a storage function $W_{i}$ for $i$ th subsystem $S_{i}$ by applying backstepping which ends with a dissipation inequality (38). Then we choose storage function $W$ for interconnected system $S$ to be the sum of all subsystem storage functions $W_{i}$ (see (44)). To get net energy dissipation for the system $S$, we dominate other subsystem interactions by the subsystem stability margins. This is done by carefully choosing the storage function $\phi_{i}$ of (7). In the special case when the interconnections are bounded by polynomials, $\phi_{i}$ is a polynomial. Thus the idea of dominance which appeared in early decentralized work ( $M$-matrix design) [15] is implemented in this nonlinear systems design.

\section{An illustrative example}

We consider the following interconnected system composed of two subsystems:

$$
\begin{aligned}
& S_{1}:\left\{\begin{array}{l}
\dot{z}_{11}=z_{12}, \\
\dot{z}_{12}=\xi_{11}+\sin (2 t) z_{21} e^{z_{21}}+\left(1+z_{11} z_{21}\right) \omega_{1}, \\
\dot{\xi}_{11}=u_{1}+\xi_{11} z_{21} \omega_{1}, \\
y_{1}=z_{11},
\end{array}\right. \\
& S_{2}:\left\{\begin{array}{l}
\dot{z}_{21}=\xi_{21}+z_{11} z_{12} \sin \left(\xi_{21}\right)+\omega_{2}, \\
\dot{\xi}_{21}=u_{2}+\omega_{2}, \\
y_{2}=z_{21} .
\end{array}\right.
\end{aligned}
$$

It can be checked that system $S$ is in form (1) and satisfies Assumptions 1 and 2. We choose the design parameters $\epsilon_{1}=1 ; \epsilon_{2}=1 ; Q_{1}=2 I ; Q_{2}=2$ to get

$$
P_{1}=\left[\begin{array}{ll}
2.8284 & 1.0000 \\
1.0000 & 1.4142
\end{array}\right], \quad P_{2}=1 .
$$

Other parameters are chosen to be $k_{i}=8 ; \delta_{i l}=\varrho_{i l}=0.1 ; \tau_{i}=1 ; c_{i}=0.1 ; d_{2 i}=2$ for $i=1,2 ; l=1,2$.

According to (52), we have

$$
\begin{aligned}
& \frac{\partial \phi_{1}}{\partial\left(z_{1}^{\mathrm{T}} P_{1} z_{1}\right)}=8+0.5 \cdot 10\left[0.5 \cdot \sqrt{\frac{\left(z_{1}^{\mathrm{T}} P_{1} z_{1}\right)+1}{0.8966}}\right]^{2}+\frac{\left(z_{1}^{\mathrm{T}} P_{1} z_{1}\right)}{1.7932} \cdot 10 \cdot\left[0.5 \cdot \sqrt{\frac{\left(z_{1}^{\mathrm{T}} P_{1} z_{1}\right)+1}{0.8966}}\right]^{4}, \\
& \frac{\partial \phi_{2}}{\partial\left(z_{2}^{\mathrm{T}} P_{2} z_{2}\right)}=8+0.5 \cdot 10 \cdot\left[e^{\sqrt{\left(z_{2}^{\mathrm{T}} P_{2} z_{2}\right)+1}}\right]^{2}+\frac{\left(z_{2}^{\mathrm{T}} P_{2} z_{2}\right)}{2} \cdot 10 \cdot\left[0.5 \cdot \sqrt{\left(z_{2}^{\mathrm{T}} P_{2} z_{2}\right)+1}\right]^{4} .
\end{aligned}
$$




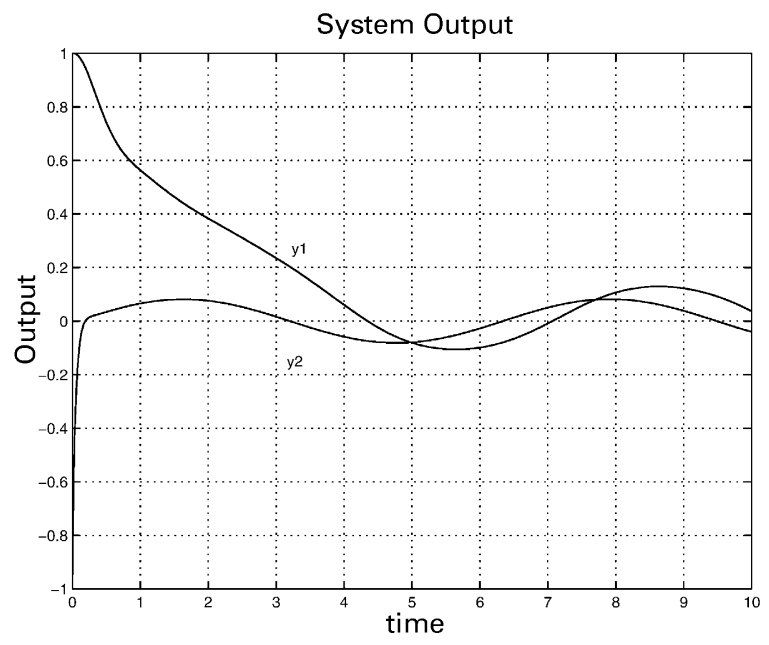

Fig. 1. Output of the closed-loop system.

Then in Step 0 by (13), we have

$$
\begin{aligned}
\xi_{11}^{*}= & -z_{11}-1.4142 z_{12}-0.5 \cdot \frac{\partial \phi_{1}}{\partial\left(z_{1}^{\mathrm{T}} P_{1} z_{1}\right)} \cdot\left(z_{11}+1.4142 z_{12}\right) \cdot 0.2 \\
& -0.1250\left(\frac{\partial \phi_{1}}{\partial\left(z_{1}^{\mathrm{T}} P_{1} z_{1}\right)}\right)^{3} \cdot\left(z_{11}+1.4142 z_{12}\right)^{3} \cdot 0.2-0.25 \frac{\partial \phi_{1}}{\partial\left(z_{1}^{\mathrm{T}} P_{1} z_{1}\right)} \cdot\left(z_{11}+1.4142 z_{12}\right), \\
\xi_{21}^{*}= & -z_{2}-0.5 \frac{\partial \phi_{2}}{\partial\left(z_{2}^{\mathrm{T}} P_{2} z_{2}\right)} \cdot z_{2} \cdot 0.2-0.1250\left(\frac{\partial \phi_{2}}{\partial\left(z_{2}^{\mathrm{T}} P_{2} z_{2}\right)}\right)^{3} \cdot z_{2}^{3} \cdot 0.2-0.25 \frac{\partial \phi_{2}}{\partial\left(z_{2}^{\mathrm{T}} P_{2} z_{2}\right)} \cdot z_{2} .
\end{aligned}
$$

Denote

$$
\begin{aligned}
& \vartheta_{1}\left(z_{1}, \xi_{11}\right)=-\frac{\partial \xi_{11}^{*}}{\partial z_{11}} z_{12}-\frac{\partial \xi_{11}^{*}}{\partial z_{12}} \xi_{11}, \\
& \vartheta_{2}\left(z_{2}, \xi_{21}\right)=-\frac{\partial \xi_{21}^{*}}{\partial z_{21}} \xi_{21}, \\
& \sigma_{10}\left(z_{1}\right)=-\frac{\partial \xi_{11}^{*}}{\partial z_{12}} \\
& \sigma_{20}\left(z_{2}\right)=-\frac{\partial \xi_{21}^{*}}{\partial z_{21}}
\end{aligned}
$$

So in Step 1 by (23) the robust decentralized control laws are constructed as

$$
\begin{aligned}
u_{1}= & -\vartheta_{1}-0.1 \cdot\left(\xi_{11}-\xi_{11}^{*}\right)-\frac{\partial \phi_{1}}{\partial\left(z_{1}^{\mathrm{T}} P_{1} z_{1}\right)}\left(z_{11}+1.4142 z_{12}\right)-0.25 \cdot \sigma_{10}^{2} \\
& -0.1250 \cdot\left(\xi_{11}-\xi_{11}^{*}\right)^{3} \cdot\left(\sigma_{10}^{4} \cdot 0.1+0.1\right) \\
u_{2}= & -\vartheta_{2}-0.1 \cdot\left(\xi_{21}-\xi_{21}^{*}\right)-\frac{\partial \phi_{2}}{\partial\left(z_{2}^{\mathrm{T}} P_{2} z_{2}\right)} z_{2}-0.25 \cdot\left(\sigma_{20}+1\right)^{2} .
\end{aligned}
$$

The system output and control input are shown in Fig. 1 and 2 which demonstrate a satisfactory disturbance attenuation performance of our closed-loop system. The initial condition used is $[1,0,0,-1,0]$ and the disturbances are $\omega_{1}=\omega_{2}=\sin (t)$. 


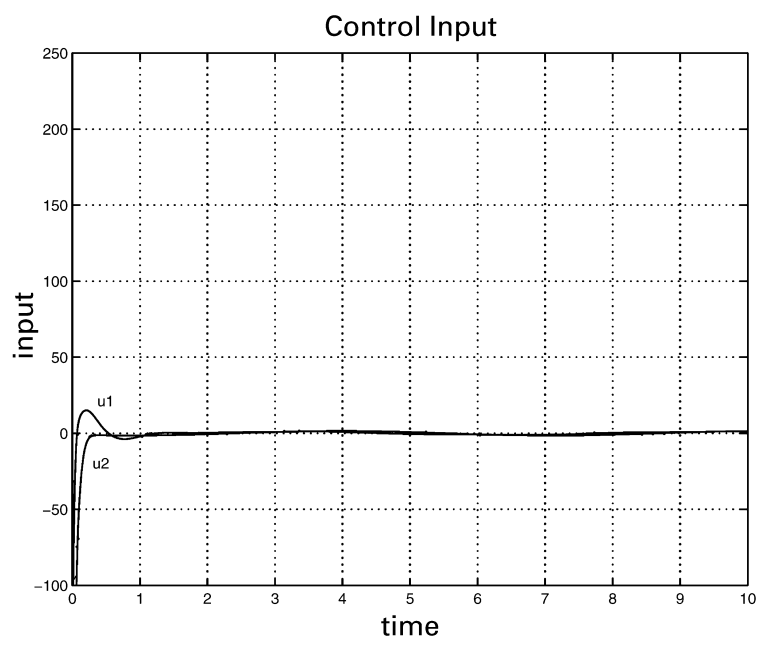

Fig. 2. Control input history.

\section{Conclusions}

The problem of decentralized $H_{\infty}$ almost disturbance decoupling has been solved for systems (1). A stepwise procedure which applied backstepping was developed and a decentralized controller was explicitly constructed. Our results allow uncertain interconnections to be time-varying and bounded by general nonlinear functions. We design a storage function that dominates the uncertainties which are not necessarily weak. The designed closed-loop system achieves arbitrary disturbance attenuation in the sense of $\mathscr{L}_{2}$ gain, and in the absence of the disturbance the system is globally uniformly asymptotically stable. Simulations exhibit a good performance of a two-subsystem example.

\section{Appendix. Proof of the claim}

As stated previously, the claim holds for system $S_{i 1}$. Assume that the claim is true for system $S_{i, l-1}$, i.e. $l=l-1$. We wish to prove the claim for $\imath=l$, i.e. system $S_{i l}$.

Consider the storage function

$$
W_{i l}=V_{i}+\sum_{k=1}^{l} \widetilde{\xi}_{i k}^{2} .
$$

Differentiating it along the solutions of the $\left(z_{i}, \xi_{i 1}, \ldots, \xi_{i l}\right)$-subsystem in (29), we have

$$
\begin{aligned}
\dot{W}_{i l} \leqslant & -\alpha_{i, l-1}\left(\left|z_{i}\right|\right)-\sum_{k=1}^{l-1} C_{i, l-1, k} \widetilde{\xi}_{i k}^{2}+\sum_{j=1}^{N} \beta_{i, l-1, j}\left(\left|z_{j}\right|\right)+\gamma_{i, l-1}\left|\omega_{i}\right|^{2} \\
& +2 \widetilde{\xi}_{i l}\left[\xi_{i, l+1}+\Xi_{i l}\left(z_{i}, \xi_{i 1}, \ldots, \xi_{i l}\right)+\Gamma_{i l}\left(t, 0, \xi_{i 1}, \ldots, \xi i l\right)+E_{i l}\left(t, 0, \xi_{i 1}, \ldots, \xi_{i l}\right) \omega_{i}\right. \\
& \left.+F_{i l}\left(t, z, \xi_{i 1}, \ldots, \xi_{i l}\right)+G_{i l}\left(t, z, \xi_{i 1}, \ldots, \xi_{i l}\right) \omega_{i}\right]
\end{aligned}
$$

where

$$
\Xi_{i l}=\frac{1}{2} \frac{\partial W_{i, l-1}}{\partial \xi_{i, l-1}}-\frac{\partial \xi_{i l}^{*}}{\partial z_{i}}\left(A_{i} z_{i}+B_{i} \xi_{i 1}\right)-\sum_{k=1}^{l-1} \frac{\partial \xi_{i l}^{*}}{\partial \xi_{i k}} \xi_{i, k+1},
$$




$$
\begin{aligned}
\Gamma_{i l} & =\sum_{k=1}^{l} \sigma_{i k} \gamma_{i k}\left(t, 0, \xi_{i 1}, \ldots, \xi_{i k}\right), \\
E_{i l} & =\sum_{k=0}^{l} \sigma_{i k} p_{i k}\left(t, 0, \xi_{i 1}, \ldots, \xi_{i k}\right), \\
F_{i l} & =\sum_{k=0}^{l} \sigma_{i k}\left[\gamma_{i k}\left(t, z, \xi_{i 1}, \ldots, \xi_{i k}\right)-\gamma_{i k}\left(t, 0, \xi_{i 1}, \ldots, \xi_{i k}\right)\right], \\
G_{i l} & =\sum_{k=0}^{l} \sigma_{i k}\left[p_{i k}\left(t, z, \xi_{i 1}, \ldots, \xi_{i k}\right)-p_{i k}\left(t, 0, \xi_{i 1}, \ldots, \xi_{i k}\right)\right]
\end{aligned}
$$

with

$$
\begin{aligned}
& \sigma_{i 0}=-\frac{\partial \xi_{i 1}^{*}}{\partial z_{i}} B_{i}, \\
& \sigma_{i k}=-\frac{\partial \xi_{i l}^{*}}{\partial \xi_{i k}} \text { when } k=1, \ldots, l-1, \\
& \sigma_{i l}=1 .
\end{aligned}
$$

To handle time-varying term $\Gamma_{i l}$, firstly we notice that the mapping

$$
\Theta:\left(z_{i}, \xi_{i 1}, \xi_{i 2}, \ldots, \xi_{i l}\right) \mapsto\left(z_{i}, \widetilde{\xi}_{i 1}, \widetilde{\xi}_{i 2}, \ldots, \widetilde{\xi}_{i l}\right)
$$

is a global diffeomorphism since its Jacobian matrix is lower triangular with all diagonal components equal to the constant one. And it preserves the origin. Therefore, $\Theta^{-1}$ is smooth and also preserves the origin. So

$$
\psi_{k}\left(\left|\left(z_{i}, \xi_{i 1}, \ldots, \xi_{i k}\right)\right|\right)=\psi_{k}\left(\left|\Theta^{-1}\left(z_{i}, \widetilde{\xi}_{i 1}, \ldots, \widetilde{\xi}_{i k}\right)\right|\right) .
$$

By a repeated application of operations used in (18), there exists a smooth function $\widetilde{\psi}_{i k}$ such that

$$
\psi_{k}\left(\left|\left(\xi_{i 1}, \ldots, \xi_{i k}\right)\right|\right) \leqslant \widetilde{\psi}_{i k}\left(z_{i}, \widetilde{\xi}_{i 1}, \ldots, \widetilde{\xi}_{i k}\right) \cdot\left(\left|z_{i}\right|+0 \sum_{l=1}^{k}\left|\widetilde{\xi}_{i l}\right|\right) \text {. }
$$

Using the bound from Assumption 2, there exists a smooth function $\Delta_{i l}(\cdot)$ such that

$$
\begin{aligned}
2 \widetilde{\xi}_{i l} \Gamma_{i l}\left(t, 0, \xi_{i 1}, \ldots, \xi_{i l}\right) \leqslant & 2\left|\widetilde{\xi}_{i l}\right|\left[\sum_{k=1}^{l}\left|\sigma_{i k}\right| \psi_{k}\left(\left|\left(\xi_{i 1}, \ldots, \xi_{i k}\right)\right|\right)\right] \\
\leqslant & 2\left|\widetilde{\xi}_{i l}\right| \sum_{k=1}^{l}\left|\sigma_{i k}\right| \widetilde{\psi}_{i k}\left(z_{i}, \widetilde{\xi}_{i 1}, \ldots, \widetilde{\xi}_{i k}\right)\left(\left|z_{i}\right|+\sum_{l=1}^{k}\left|\widetilde{\xi}_{i l}\right|\right) \\
& \leqslant\left[\frac{1}{d_{1 i}} \widetilde{\xi}_{i l}^{2}\left(\sum_{k=1}^{l} \sigma_{i k} \widetilde{\psi}_{i k}\right)^{2}+d_{1 i}\left|z_{i}\right|^{2}\right]+\left[\widetilde{\xi}_{i l}^{2} \Delta_{i l}\left(z_{i}, \widetilde{\xi}_{i 1}, \ldots, \widetilde{\xi}_{i l}\right)+\sum_{k=1}^{l-1} d_{3 i}\left|\widetilde{\xi}_{i k}\right|^{2}\right] \\
& \triangleq \widetilde{\xi}_{i l}^{2} \widetilde{\Delta}_{i l}\left(z_{i}, \widetilde{\xi}_{i 1}, \ldots, \widetilde{\xi}_{i l}\right)+\left[d_{1 i}\left|z_{i}\right|^{2}+\sum_{k=1}^{l-1} d_{3 i}\left|\widetilde{\xi}_{i k}\right|^{2}\right]
\end{aligned}
$$

where $d_{3 i}$ is a positive design parameter whose rule is given in (43). 
Similarly, apply the bounds from Assumption 2 and 1 to the last three terms of (69),

$$
\begin{aligned}
2 \widetilde{\xi}_{i l} E_{i l}\left(t, 0, \xi_{i 1}, \ldots, \xi_{i l}\right) \omega_{i} \leqslant & 2\left|\widetilde{\xi}_{i l}\right|\left(\sum_{k=0}^{l}\left|\sigma_{i k}\right| \Psi_{i k}\left(\left|\left(\xi_{i 1}, \ldots, \xi_{i k}\right)\right|\right)\right)\left|\omega_{i}\right| \\
& \leqslant \frac{1}{d_{2 i}}\left|\widetilde{\xi}_{i l}\right|^{2}\left[\sum_{k=0}^{l}\left|\sigma_{i k}\right| \Psi_{i k}\left(\left|\left(\xi_{i 1}, \ldots, \xi_{i k}\right)\right|\right)\right]^{2}+d_{2 i}\left|\omega_{i}\right|^{2}, \\
2 \widetilde{\xi}_{i l} F_{i l}\left(t, z, \xi_{i 1}, \ldots, \xi_{i l}\right) \leqslant & 2\left|\widetilde{\xi}_{i l}\right|\left(\sum_{k=0}^{l}\left|\sigma_{i k}\right| \sum_{j=1}^{N} a_{i k j}\left(\left|\left(\xi_{i 1}, \ldots, \xi_{i k}\right)\right|\right) \varphi_{i k j}\left(\left|z_{j}\right|\right)\right) \\
\leqslant & \widetilde{\xi}_{i l}^{2} \sum_{k=0}^{l} \sum_{j=1}^{N} \sigma_{i k}^{2} \delta_{j k} a_{i k j}^{2}\left(\left|\left(\xi_{i 1}, \ldots, \xi_{i k}\right)\right|\right)+\sum_{k=0}^{l} \sum_{j=1}^{N} \delta_{j k}^{-1} \varphi_{i k j}^{2}\left(\left|z_{j}\right|\right), \\
2 \widetilde{\xi}_{i l} G_{i l}\left(t, z, \xi_{i 1}, \ldots, \xi_{i l}\right) \omega_{i} \leqslant & 2\left|\widetilde{\xi}_{i l}\right|\left(\sum_{k=0}^{l}\left|\sigma_{i k}\right| \sum_{j=1}^{N} b_{i k j}\left(\left|\left(\xi_{i 1}, \ldots, \xi_{i k}\right)\right|\right) \varphi_{i k j}\left(\left|z_{j}\right|\right)\right) \omega_{i} \\
\leqslant & \frac{1}{4 \tau_{i}^{2}} \widetilde{\xi}_{i l}^{4} \sum_{k=1}^{l} \sum_{j=1}^{N} \sigma_{i k}^{4} \varrho_{j k} b_{i k j}^{4}\left(\left|\left(\xi_{i 1}, \ldots, \xi_{i k}\right)\right|\right)+\sum_{k=0}^{l} \sum_{j=1}^{N} \varrho_{j k}^{-1} \Phi_{i k j}^{4}\left(\left|z_{j}\right|\right) \\
& +(l+1) N \tau_{i}\left|\omega_{i}\right|^{2} .
\end{aligned}
$$

So we choose virtual controller $\xi_{i, l+1}=\xi_{i, l+1}^{*}$ as

$$
\begin{aligned}
\xi_{i, l+1}^{*}= & -\frac{1}{2}\left\{c_{i l} \widetilde{\xi}_{i l}+2 \Xi_{i l}+\widetilde{\xi}_{i l} \widetilde{\Delta}_{i l}\left(z_{i}, \widetilde{\xi}_{i 1}, \ldots, \widetilde{\xi}_{i l}\right)+\frac{1}{d_{2 i}} \widetilde{\xi}_{i l}\left[\sum_{k=0}^{l}\left|\sigma_{i k}\right| \Psi_{i k}\left(\left|\left(\xi_{i 1}, \ldots, \xi_{i k}\right)\right|\right)\right]^{2}\right. \\
& \left.+\widetilde{\xi}_{i l} \sum_{k=0}^{l} \sum_{j=1}^{N} \sigma_{i k}^{2} \delta_{j k} a_{i k j}^{2}\left(\xi_{i 1}, \ldots, \xi_{i k}\right)+\frac{1}{4 \tau_{i}^{2}} \widetilde{\xi}_{i l}^{3} \sum_{k=1}^{l} \sum_{j=1}^{N} \sigma_{i k}^{4} \varrho_{j k} b_{i k j}^{4}\left(\xi_{i 1}, \ldots, \xi_{i k}\right)\right\},
\end{aligned}
$$

where $c_{i l}$ is a positive constant.

Substituting (82) into (69) and in view of bounds (78)-(81), we get

$$
\begin{aligned}
\dot{W}_{i l} \leqslant & -\left[\alpha_{i, l-1}\left(\left|z_{i}\right|\right)-d_{1 i}\left|z_{i}\right|^{2}\right]-\left[\sum_{k=1}^{l-1}\left(C_{i, l-1, k}-d_{3 i}\right) \widetilde{\xi}_{i k}^{2}+c_{i l} \widetilde{\xi}_{i l}^{2}\right] \\
& +\sum_{j=1}^{N}\left[\beta_{i, l-1, j}\left(\left|z_{j}\right|\right)+\sum_{k=0}^{l}\left(\delta_{j k}^{-1} \varphi_{i k j}^{2}\left(\left|z_{j}\right|\right)+\varrho_{j k}^{-1} \Phi_{i k j}^{4}\left(\left|z_{j}\right|\right)\right)\right]+\left[\gamma_{i, l-1}+(l+1) N \tau_{i}+d_{2 i}\right]\left|\omega_{i}\right|^{2} .
\end{aligned}
$$

With appropriate definitions of $\alpha_{i l}, C_{i l k}, \beta_{i l j}$ and $\gamma_{i l}$, we get (31) with $l=l$ which completes the proof of the Claim.

\section{References}

[1] Y.H. Chen, G. Leitmann, Z.K. Xiong, Robust control design for interconnected systems with time-varing uncertainties, Int. J. Control 54 (5) (1991) 1119-1142.

[2] C.F. Cheng, Disturbances attenuation for interconnected systems by decentralized control, Int. J. Control 66 (2) (1997) $213-224$. 
[3] D.T. Gavel, D.D. Šiljak, Decentralized adaptive control: structural conditions for stability, IEEE Trans. Automat. Control 34 (4) (1989) 413-426.

[4] M.C. Han, Y.H. Chen, Decentralized control design: uncertain systems with strong interconnections, Int. J. Control 61 (6) (1995) 1363-1385.

[5] D.J. Hill, C. Wen, G.C. Goodwin, Stability analysis of decentralised robust adaptive control, System Control Lett. 11 (1988) $277-284$.

[6] P. Ioannou, Decentralized adaptive control of interconnected systems, IEEE Trans. Automat. Control 31 (4) (1986) $291-298$.

[7] A. Isidori, Nonlinear Control Systems, in Series of Communications and Control Engineering, third ed., Springer, London, 1995.

[8] A. Isidori, A note on almost disturbance decoupling for nonlinear minimum phase systems, Syst. Control Lett. 27 (1996) $191-194$.

[9] S. Jain, F. Khorrami, Decentralized adaptive control of a class of large-scale interconnected nonlinear systems, IEEE Trans. Automat. Control 42 (2) (1997) 136-154.

[10] D.C. Jiang, Z.P. Jiang, $H_{\infty}$ almost disturbance decoupling with stability for uncertain nonlinear systems, Proceedings of the fourth European Control Conference, Brussels, Belgium, 1997.

[11] Z.P. Jiang, A.R. Teel, L. Praly, Small-gain theorem for ISS systems and applications, Math. Control, Signals Systems 7 (1994) 95-120.

[12] M. Krstic, I. Kanellakopoulos, P. Kokotovic, Nonlinear Adaptive Control Design, Wiley, New York, 1995.

[13] I.M.Y. Mareels, D.J. Hill, Monotone stability of nonlinear feedback systems, J. Math. Systems, Estimation Control 2 (3) (1992) 275-291.

[14] R. Marino, W. Respondek, A.J. van der Schaft, P. Tomei, Nonlinear $H_{\infty}$ almost disturbance decoupling, Systems Control Lett. 23 (1994) 159-168.

[15] P.J. Moylan, D.J. Hill, Stability criteria for large-scale systems, IEEE Trans. Automat. Control 23 (2) (1978) $143-149$.

[16] R. Ortega, A. Herrera, A solution to the decentralized adaptive stabilization problem, Proceedings of the 31st IEEE Conference on Decision and Control, Tucson, Arizona, 1992, pp. 3622-3627.

[17] L. Shi, S.K. Singh, Decentralized adaptive controller design for large-scale systems with higher order interconnections, IEEE Trans. Automat. Control 37 (8) (1992) 1106-1118.

[18] L. Shi, S.K. Singh, Decentralized control for interconnected uncertain systems: extensions to higher-order uncertainties, Int. J. Control 57 (6) (1993) 1453-1468.

[19] D.D. Šiljak, Large-scale dynamic systems: stability and structure, Series of System Science and Engineering, North-Holland, New York, 1978.

[20] A.J. van der Schaft, $L_{2}$-gain analysis of nonlinear systems and nonlinear state feedback $H_{\infty}$ control, IEEE Trans. Automat. Control 37 (1992) 770-784.

[21] C. Wen, Direct decentralized adaptive control of interconnected systems having arbitrary subsystem relative degrees, Proceedings of the 33rd IEEE Conference on Decision and Control, Lake Buena Vista, FL, 1994, pp. 1187-1192.

[22] C. Wen, Y.C. Soh, Decentralized adaptive control using integrator backstepping, Automatica 33 (1997) 1719-1724.

[23] J.C. Willems, Dissipative dynamical systems - Part 1: general theory, Arch. Rot. Mech. Anal. 45 (1972) 321-351.

[24] G. Zhai, K. Yasuda, M. Ikeda, Decentralized $H_{\infty}$ control of large-scale systems via output feedback, Proceedings of the 32nd IEEE Conference on Decision and Control, San Antonio, Texas, 1993, pp. 1652-1653. 